\title{
Secondary Attachments and Adolescent Self Concept ${ }^{1}$
}

\author{
Carolyn Adams-Price \\ Institute of Gerontology, University of Michigan
}

\author{
A. L. Greene ${ }^{2}$ \\ West Virginia University
}

This study examined the popular notion that crushes or secondary attachments to celebrity figures are an important aspect of self-concept development during adolescence. In a repeated measures design, 79 male and female 5th, 8th, and 11th graders and college sophomores completed a set of personality scales, first describing themselves and later, describing the favorite celebrity. Repeated measures multivariate analyses of variance (MANOVA) analysis of self-object congruence revealed no significant main or interaction effects for the type of attachment, gender, or age of subject. Significant within subject effects were obtained for the repeated measures factor (self-object congruence). Overall, students perceived their attachment objects to be more agentic, yet less expressive and emotionally vulnerable than themselves. MANOVA analyses indicated that males and older students perceived their attachment objects to be higher in agency than expressivity, whereas females and younger students perceived their attachment objects to be higher in expressivity than agency. These data indicate that the function served by secondary attachments in the development of self-concept may be quite similar for adolescent males and females.

\footnotetext{
'This research was supported by a grant awarded to the second author ("Transitions in adolescence") by the National Research Council. This paper is an expanded version of one presented at the biennial meeting of the Society for Research on Child Development, Baltimore, MD, April 26-28, 1987. The authors gratefully acknowledge the assistance of David Grimm and Paul Klaczynski in the collection and analysis of data presented here, and Sharon Foster for her comments on a previous version of the manuscript.

${ }^{2}$ To whom correspondence should be addressed at Department of Psychology, P.O. Box 6040, West Virginia University, Morgantowm, WV 26506-6040.
} 
Crushes are a popular topic in the literature on adolescence (Davidson, 1973; Hebdige, 1979; Landis, 1970). Defined as an unreciprocated or unarticulated attachment to another, crushes are generally characterized by the frequent occurrence of fantasies in which the personal qualities of the other are greatly enhanced or idealized (Fromm, 1967). Adolescent crushes are best conceptualized as "secondary attachments"; that is, feelings or imagined relationships that are formed for distant figures or objects. ${ }^{3}$ Such attachments need not be romantic in nature, nor indeed, their object aware of the fantasies that it generates. Unlike mature relationships, the direction of affective influence is presumed to be unilinear rather than reciprocal (i.e., from the adolescent to the attachment object): Indeed, as posited by psychoanalytic theory (Erikson, 1950; Freud, 1925), the absence of reciprocity or even proximity is a fundamental aspect of the object's phenomenological appeal.

Seen in this manner, secondary attachments are not too dissimilar than the imaginary friendships of childhood (Benson, 1980; Klein, 1985). Both involve the construction of an imaginary relationship characterized by warmth and understanding (Fraiburg, 1968; Fromm, 1967; Landis, 1970; Manosevitz, Prentice, \& Wilson, 1973; Somers \& Yawhey, 1980). Adolescents, however, are not thought to invent the attachment object, as do children, but to project their own feelings, even personality characteristics to it (Fromm, 1967; Landis, 1970).

Sufficient consensual and anecdotal evidence exists regarding secondary attachments to warrant their examination (Davidson, 1973; Hebdige, 1979). Yet this aspect of adolescence has been largely overlooked in the empirical literature. In part, this oversight may be due to researchers' concerns about the apparent pursuit of cultural fads which may not be reflective of stable developmental patterns. Such research need not, however, focus on popular figures, per se, but on the common features of secondary attachments, regardless of who the popular figure of the moment happens to be. Further, sociohistorical changes in popular celebrities may reflect underlying changes in cultural norms and mores that merit attention. The successive popularities of Sinatra, the Beatles, and the Grateful Dead may be seen as reflective of the corresponding social changes which characterized those periods, for example. ${ }^{4}$ By failing to examine the phenomenon of secondary

\footnotetext{
${ }^{3}$ We use the term object, following object relations theory (Winnicott, 1965, 1971). In so doing, we assume the object to be an internalized representation of a significant other. That representation, although highly salient to the individual, may show little veridicality with the actual characteristics of the other. Yet it is that internal representation, rather than the object itself, which titrates the development of sexuality and self-concept in this respect. Hereinafter, therefore, we shall make use of the terms secondary attachment, rather than crush, and attachment object, rather hero or idol, to underscore this distinction.

${ }^{4}$ For an interesting discussion of these and related issues, see Boxer, Gershonson, and Offer (1984).
} 
attachments empirically, psychologists have overlooked a unique opportunity to study the normative development of adolescent sexuality and self-concept. This point is perhaps best illustrated by considering the role attributed to adolescent crushes by Erikson $(1950,1968)$ and Freud (1925).

In Eriksonian theory (Erikson, 1950, 1968), the primary task of adolescence is the consolidation of ego identity. An important aspect of that process is the adolescent's experimentation with novel roles and identities (Marcia, 1966, 1980). The role confusion endemic to that process is thought to lead to temporary overidentifications with heroes as one means of role experimentation (e.g., assuming the dress and mannerisms of the figure). Such overidentification may be individual or shared among members of a particular group - in the context of which it also provides both a focal image and a mechanism of social exchange (Elkind, 1980; Erikson, 1950). Erikson (1968) further suggests that one function served by secondary attachments is that they permit the safe exploration of possible roles and identities. For males, a central aspect of this process is the search for occupational identity. By experimenting with the fantasied characteristics and roles of popular figures, the adolescent male is able to explore and so discover the lineaments of his own identity. Females, for whom identity consolidation is held to occur later, through committed relationships (Erikson, 1968), obtain similar purpose in secondary attachments. By experimenting in fantasied romances with popular figures, females may began to delineate the outlines of a future partner. Theoretically at least, secondary attachments provide a safe context in which both male and female adolescents can experiment with alternate identities in the search for a consolidated sense of self (Erikson, 1950).

Traditional Freudian theory also implies a purpose to adolescent crushes, although the primary emphasis is on sexual, rather than social, identity development (Freud, 1925). Crushes are seen to be a normative aspect of sexual development during adolescence ("the sexual life of maturing youth is almost entirely restricted to indulging in fantasies"; Freud, 1925, p. 266). That is, heightened libidinal urges that cannot be directed toward proximal objects, such as parents or peers, can be safely transferred to more distal ones, such as celebrity figures. As the prospect of real sexual attachment may be too frightening, the young adolescent projects libidinal attachments to safe, albeit distant, objects (Freud, 1925; Fromm, 1967; Muus, 1980). Secondary attachments thus may serve an important function in the development of mature sexuality by permitting exploration and experimentation at a safe remove from immediate experience and immediate consequence (Freud, 1925; Fromm, 1967; Landis, 1970).

Several hypotheses follow from these formulations. First, two types of secondary attachment should be evident in adolescents' descriptions of a favorite object or celebrity figure: (a) attachments based on romantic or sexual feelings for the object; and (b) attachments based on an identification 
with, or a desire to be like, the object. Second, these attachments should differentially associate with gender such that the frequency of identificatory attachments should be higher among males and the frequency of romantic attachments higher among females. Third, objects of romantic and identificatory attachment should hold certain (attributed) characteristics in common: (a) attachment objects should be "larger than life" figures, endowed with suitably heroic qualities; (b) attachment objects should be similar (i.e., identificatory attachments) or complementary (i.e., romantic attachments) to the adolescent's sense of self; and (c) adolescents should experience both types of attachments in similar fashion, spending time thinking about the object or discussing the object with friends.

As part of a larger investigation of adolescent self-concept development, the present study examined the degree of congruence between the attributed characteristics of secondary attachment objects and the self-described personality characteristics of young and older adolescents (self-object congruence).

\section{METHOD}

\section{Sample}

Seventy-nine Caucasian students participated in this study: 10 male and 10 female 5th graders ( $X$ age $=10.55$ years), 11 male and 8 female 8th graders ( $X$ age $=13.37$ years), 10 male and 10 female 11th graders $(X$ age $=$ 16.20 years), and 10 male and 10 female college sophomores $(X$ age $=19.25$ years). Elementary, junior, and senior high school students were selected from the 5th-, 8th-, and 11th-grade college preparatory curriculum at their respective schools. College sophomore participants were selected from a volunteer pool of students then enrolled in an introductory psychology course at the local university. The sample was predominantly middle class, as determined by the Dunkin Index (Reiff, 1961).

\section{Instruments}

Data concerning secondary attachments were obtained through the use of a self-administered questionnaire, which included the Spence and Helmreich (1978) Personality Attributes Questionnaire (PAQ). Questionnaires requested the student to describe an attachment object ("Who is your favorite celebrity?"), including the kind of relationship that they would like to have with that object ("Imagine that there was a magic wand that could suddenly transform you into a person close to your favorite celebrity and you could choose your relationship. Who would you most like to be?") and the 
amount of time spent in various activities related to the object (e.g., "How often do you think about your favorite celebrity?" "How often do you talk about your favorite celebrity with your best friend? With other friends?").

The Spence and Helmreich (1978) PAQ was used to measure the personality characteristics of each participant, as well as the personality characteristics attributed to the attachment object. The PAQ consisted of 24 items, divided into three subscales (Masculinity, Femininity, and Masculinity/Femininity). Each PAQ subscale consisted of eight personality traits, presented in five-point bipolar format $(1=$ very passive; $5=$ very active $)$. Items comprising the Masculinity (M) subscale consisted of personality attributes thought to be socially desirable in both sexes, but more commonly occurrent among males (e.g., independence). High scores on the M subscale reflected more masculine characteristics such as agency and instrumentality; low scores reflected more feminine characteristics. Femininity (F) subscale items represented personality attributes thought to be socially desirable in both sexes, but more commonly occurrent among females (e.g., gentle). High scores on this subscale reflected more feminine characteristics, such as expressivity, whereas low scores reflected more masculine characteristics. Finally, Masculinity/Femininity (MF) subscale items consisted of attributes that varied in social desirability for either sex (e.g., submissiveness for females and dominance for males). High scores on the MF scale reflected more "feminine" characteristics; lower scores reflected more "masculine" characteristics (Spence \& Helmreich, 1978).

\section{Procedure}

Participants were tested in small groups of 5-10. Following a brief description of the purpose and procedures of the study, each student was given a protocol containing the instruments described above. In a repeated measures design, students were instructed to complete the PAQ, describing first themselves and, later in the protocol, their attachment objects. Students typically required 45 minutes to 1 hour to complete the protocol. At the conclusion of each test session, students were reimbursed for their participation.

\section{Measures}

Type of secondary attachment was determined by student's responses to one of five response choices in the "magic wand" question: (a) identificatory (i.e., be the celebrity), (b) romantic (i.e., be the celebrity's girlfriend/boyfriend), (c) platonic (i.e., be the celebrity's friend), (d) relational (i.e., be the celebrity's relative or child), and (e) other. 
Three measures of personality were obtained for each student and his/ her attachment object: (a) Instrumentality, as measured by the respondent's (and object's) mean score on the Masculinity (M) subscale; (b) Expressivity, as measured by the respondent's (and object's) mean score on the Femininity (F) subscale; and (c) Vulnerability, as measured by the respondent's (and object's) mean score on the Masculinity/Femininity (MF) subscale (Spence \& Helmreich, 1978).

\section{RESULTS}

Data analyses were conducted in sequential fashion to examine (a) the types of secondary attachment to a favorite celebrity endorsed by the sample, (b) the personality characteristics attributed to the object of that attachment, (c) the degree of congruence between adolescents' self-perceptions and their perceptions of the attachment object, and (d) the salience of secondary attachments in adolescents' daily experience.

\section{Categories of Adolescents' Secondary Attachments}

Table I presents the distribution of secondary attachment categories, overall and by gender. Of the five possible categories, only two, identificatory and romantic attachments, received marked endorsement by the sample: $30 \%$ of the students endorsed the wish to "be the celebrity" and $25 \%$ endorsed the wish to be the celebrity's romantic partner or spouse. As romantic and identificatory attachments were the two predicted categories and as these categories captured the majority of responses, subsequent analyses included only these two.

Chi-square analyses revealed significant gender differences in the type of attachment preferred. Whereas females $(39.5 \%)$ preferred romantic attachments ("would like to be the star's girlfriend/wife"), males (36.6\%) preferred identificatory attachments ("would like to be the celebrity") $\left[\chi^{2}(4, N\right.$ $=44)=10.08, p<.05$ ]. However, grade was unrelated to the type of attachment preferred $\left[\chi^{2}(3, N=44)=1.51, \mathrm{~ns}\right]$.

\section{Characteristics of the Attachment Object}

Both males and females overwhelmingly identified male celebrity figures as attachment objects $\left[\chi^{2}(1, N=44)=30.51, p<.001\right]$. Analysis of variance (ANOVA) indicated significant main effects of gender and grade in the perceived age of the attachment object. Males described significantly 
Table I. Percent Distribution of Endorsement for Secondary Attach-

\begin{tabular}{lccc}
\multicolumn{4}{c}{ ments, Overall and by Gender } \\
\hline & \multicolumn{3}{c}{ \% Endorsement } \\
\cline { 2 - 4 } & All & Males & Females \\
Attachment responses & $(N=79)$ & $(N=41)$ & $(N=38)$ \\
\hline Be the celebrity & 30.4 & 36.6 & 23.7 \\
$\begin{array}{l}\text { Be the celebrity's roman- } \\
\text { tic partner }\end{array}$ & 25.3 & 12.2 & 39.5 \\
Be the celebrity's friend & 16.5 & 17.1 & 15.8 \\
Be the celebrity's relative & 15.2 & 14.6 & 15.8 \\
Other & 12.7 & 19.5 & 5.2 \\
\hline${ }^{a} \chi^{2}(4, N=80)=10.08, p<.05$.
\end{tabular}

older attachment objects than those described by females $[F(1,43)=28.28$, $p<.001]$ and younger adolescents described significantly younger attachment objects than those identified by older adolescents $[F(3,43)=7.25, p$ $<.001]$. However, no significant Gender $\times$ Age Group interaction effects were obtained $[F(3,36)=0.11, \mathrm{~ns}]$.

Repeated measures multivariate analysis of variance (MANOVA) indicated no significant grade $[F(3,35)=0.94$, ns $]$ or gender $[F(1,35)=$ $0.03, \mathrm{~ns}]$ effects in personality characteristics attributed to the attachment object (Masculinity vs. Femininity vs. Masculinity/Femininity). However, a significant main effect was obtained for the repeated measures factor (object personality) $[F(2,70)=44.87, p<.001]$. Overall, students perceived their attachment objects to be higher in agency than expressivity $[F(1,35)=$ $36.52, p<.001]$ and higher in expressivity than emotional vulnerability, $[F(1,35)=55.31, p<.001]$.

Significant Grade $\times$ Object Personality $[F(6,70)=2.93, p<.02]$ and Gender $\times$ Object Personality $[F(2,70)=3.56, p<.04]$ interactions also were obtained. Table II presents the mean object PAQ scores and standard deviations obtained in that analysis. Whereas males perceived their attachment objects to be higher in agency than expressivity, females perceived their attachment objects to be higher in expressivity than agency $[F(1,35)=$ $6.31, p<.01]$. Further, females perceived their attachment objects to be more wordly ("knows more about life") than males $[F(4,35)=3.39, p<$ .02]. As well, older students perceived their objects to be higher in agency than expressivity, whereas younger students perceived their objects to be higher in expressivity than agency $[F(3,35)=4.20, p<.02]$. No grade $[F(3,35)=2.64 \mathrm{~ns}]$ or attachment type $[F(1,40)=2.34$, ns $]$ effects were obtained in perceived worldliness.

Further, no effects of attachment type (identificatory vs. romantic) in the objects' attributed personally characteristics were obtained $[F(2,78)=$ 0.05 , ns]. However, objects of identificatory attachments were seen as signi- 
Table II. Mean PAQ Subscale Scores and Standard Deviations for Secondary Attachment Objects, by Grade and Subject Gender

\begin{tabular}{lccc}
\hline & \multicolumn{3}{c}{ PAQ subscales } \\
\cline { 2 - 4 } \multicolumn{1}{c}{ Group } & Masculinity & Femininity & $\begin{array}{c}\text { Masculinity/ } \\
\text { Femininity }\end{array}$ \\
\hline \multicolumn{4}{c}{ Grade level } \\
5th & $3.34(.53)$ & $3.83(.66)$ & $3.11(.80)$ \\
8th & $3.36(.58)$ & $3.57(.65)$ & $2.75(.65)$ \\
11th & $3.78(.49)$ & $3.44(.59)$ & $2.30(.67)$ \\
College & \multicolumn{4}{c}{} \\
sophomore & $3.76(.52)$ & $3.75(.98)$ & $2.34(.79)$ \\
\multicolumn{4}{c}{ Gender } \\
Females & $3.30(.45)$ & $3.74(.76)$ & $2.81(.70)$ \\
Males & $3.83(.54)$ & $3.53(.63)$ & $2.46(.84)$ \\
\hline
\end{tabular}

ficantly more attractive than objects of romantic attachments $[F(1,41)=$ $5.96, p<.02]$.

\section{Self-Object Congruence}

Repeated measures MANOVA analysis revealed significant effects of the repeated measures factor (self-object congruence) in Masculinity $[F(1$, $35)=9.12, p<.005]$, Femininity $[F(1,35)=5.99, p<.02]$, and Masculinity/Femininity $[F(1,35)=4.44, p<.04]$ subscale scores. Regardless of gender or grade, students perceived their attachment objects to be more agentic, yet less expressive and less emotionally vulnerable than themselves.

However, no significant main effects of attachment type in self-object congruence were obtained $[F(2,81)=0.64$, ns]. Nor were significant gender or grade differences obtained in self-object congruence in Masculinity $[F(3,35)=1.10$, ns, and $F(1,35)=0.84$, ns, respectively $]$, Femininity $[F(3$, $35)=0.12$, ns, and $F(1,35)=0.62$, ns, respectively], or Masculinity/Femininity $[F(3,35)=0.99$, ns, and $F(1,35)=0.52$, ns, respectively $]$.

\section{Salience of Secondary Attachments}

In the final examination of these data, one-way ANOVAs were performed to examine the salience of secondary attachments (e.g., the amount of time spent thinking about the object of discussing the object with 
Table III. Mean PAQ Subscale Scores and Standard Deviations for Self and Secondary Attachment Object

\begin{tabular}{lccc}
\hline PAQ subscales & Self & Object & $F$ \\
\hline Masculinity & $3.52(.60)$ & $3.84(.62)$ & $9.12^{c}$ \\
Femininity & $3.91(.56)$ & $3.64(.70)$ & $5.99^{b}$ \\
Masculinity/ & & & \\
$\quad$ Femininity & $2.92(.76)$ & $2.65(.78)$ & $4.44^{a}$ \\
\hline${ }^{a} p<.05$. & & & \\
${ }^{b} p<.02$. & & & \\
${ }^{c} p<.001$. & & &
\end{tabular}

friends). ${ }^{5}$ Age, gender, and attachment type differences were obtained for only two of the salience variables considered: (a) younger adolescents discussed the attachment object with their mothers more frequently than older adolescents $[F(3,35)=4.26, p<.05]$, (b) females discussed the attachment with their mothers more frequently than males $[F(1,35)=3.36, p<.05]$, and (c) adolescents who preferred romantic attachments were more likely to collect paraphernalia associated with the object than were students who preferred identificatory attachments $\left[\chi^{2}(1, N=44)=4.94, p<.05\right]$.

\section{DISCUSSION}

The present findings provided support for the suggestion that secondary attachments to distant figures play a role in the development of social and sexual identity during adolescence. Two forms of secondary attachment predominated in the fantasied relationships described by the sample: (a) romantic attachments, in which adolescents endorsed a desire to be the object's romantic partner; and (b) identificatory attachments, in which adolescents endorsed a desire to be the object. However, the common assumption that secondary attachments are primarily characteristic of female rather than male adolescents (Erikson, 1950; Landis, 1970) was not supported. Both males and females easily described fantasied relationships with distant objects.

The gender differences that did emerge in the present study are congruent with previous research on adolescent sexuality (Gagnon, 1977; Langfeldt, 1980; Landis, 1970; Matteson, 1975). Previous investigators have suggested that the early sexual fantasies of adolescent females are thought to be

\footnotetext{
${ }^{5}$ As the obtained cell sizes were too small to permit multivariate analysis of these data, a series
} of one-way ANOVAs was performed. 
more romantic in character, with greater emphasis given to the mental attributes and personality characteristics of the fantasied object, and less to its physical endowment (Davis, 1968; Gagnon, 1977; Langfeldt, 1980). That adolescent females in the present study almost uniformly preferred romantic to identificatory attachments may be seen as a predictable consequence of differences in early gender socialization experiences; that adolescent males preferred identificatory attachments to agentic, highly "masculinized" objects can be interpreted in similar fashion (Gilligan, 1982). Thus, it is arguable that secondary attachments may serve comparable functions for adolescent males and females by facilitating the maintenance of appropriate, if traditional, gender role attitudes and behavior (Elkind \& Bowen, 1979; Erikson, 1950, 1968). What appears to differ, however, is the configuration that the attachment assumes.

Both the type of attachment preferred and the personality characteristics attributed to attachment objects were more finely drawn along the lines of gender than the fact of the attachment itself. Males preferred identificatory secondary attachments, whereas females preferred romantic secondary attachments. Further, males described objects who were older and more agentic than those described by females. These findings are particularly noteworthy as many students identified the same male figure as an attachment object. This difference in the configuration of even fantasied relationships parallels the distinction drawn by other authors between the socialization of females toward greater relatedness and of males towards greater instrumentality (Erikson, 1950, 1968; Gilligan, 1982; Spence \& Helmreich, 1978).

The grade differences obtained in characteristics attributed to the attachment object, however, are less easily accommodated by a hypothesis of differential socialization. That younger students perceived the attachment object to be higher in expressivity and nurturance may reflect their comparatively greater need for affective security. This interpretation is implicit in both psychoanalytic and object relations theory, each of which assumes the utility of distant figures in the resolution of libidinal object ties (Freud, 1925; Winnicott, 1971).

The prediction of self-object congruence was not supported by the present findings. Regardless of grade, gender, or type of secondary attachment, objects were perceived to be more agentic, yet less expressive and less vulnerable than adolescents saw themselves. The stereotypic object that emerges from these data is not quite the "larger than life" figure described by theory (Erikson, 1950, 1968), but certainly one that is more powerful than the adolescent.

Like all first efforts, the questions generated by the present findings are more provocative than the questions addressed. For example, although the decision to focus on the two attachment types predicted by theory (i.e., 
romantic and identificatory attachments) captured the modal response $(55 \%)$, alternate forms of secondary attachment remain unexplored. Correspondingly, the similarly between secondary attachments to distant objects and secondary attachments to more proximal ones, such as teachers or peers, remains unclear. We might, however, anticipate that proximal attachments would be more sexualized than distant ones (Freud, 1925; Fromm, 1967). Finally, our attempts to examine the object's salience overlooked an important aspect of the attachment experience - the intensity of affect associated with the object itself. Objects for whom adolescents experience intense affect or stronger attachment may sharply differ in attributed personality characteristics, as well as salience, from objects for whom adolescents experience only moderate attachment. Further investigations directed at disentangling these aspects are necessary.

The findings of this study suggest that secondary attachments are an important, if overlooked, aspect of adolescent self-concept. By no means are such attachments the only or the most critical venue of identity formation, but as shown here, they remain an important aspect of its development. It is hoped that these findings will encourage future investigations in this area.

\section{REFERENCES}

Benson, R. M. (1980). Narcissistic guardians: Developmental aspects of transitional objects, imaginary companions and career fantasies. Adolescent Psychiatry, 8, 47-89.

Boxer, A. M., Gershonson, H. P., \& Offer, D. (1984). Historical time and social change in the adolescent experience. In D. Offer, E. Ostrov, \& K. I. Howard (Eds.), New directions for mental health services: Patterns of adolescent self-image. San Francisco: JosseyBass.

Davidson, S. (1973). Feeding on dreams in a bubble gum culture. Atlantic Monthly, 232, $62-66$.

Davis, M. (1968). Sex and the adolescent. New York: Dial Press.

Elkind, D. (1967). Egocentricism in adolescence. Child Development, 38, 1025-1034.

Elkind, D. (1980). Strategic interactions in early adolescence. In J. Adelson (Ed.), Handbook of adolescent psychology. New York: John Wiley \& Sons.

Elkind, D. \& Bowen, R. (1979). Imaginary audience behavior in children and adolescents. Developmental Psychology, 15, 38-44.

Erikson, E. (1950). Childhood and society. New York: Norton.

Erikson, E. (1968). Identity: Youth and Crisis. New York: W. W. Norton \& Company.

Fraiberg, S. (1968). The magic years. New York: Scribner.

Freud, S. (1925). Three contributions to the sexual theory. Nervous and mental disease monograph series (No. 7). New York: Nervous and Mental Disease Publishing.

Fromm, A. (1967). Ability to love. New York: Farrar, Strass, \& Giroux.

Gagnon, J. (1977). Human sexualities. Glenview, IL: Scott, Foresman.

Gilligan, C. (1982). In a different voice: Psychological theory and women's development. Cambridge, MA: Harvard University Press.

Hebdige, D. (1979). Subculture: The meaning of style. London: Methuen.

Klein, B. R. (1985). A child's imaginary companion: A transitional self. Clinical Social Work, $13,272-282$.

Landis, C. (1970). Sex in development. College Park, MD: McGrath. 
Langfeldt, T. (1980). Child sexuality: Development and problems. In J. Samson (Ed.), Childhood and sexuality: Proceedings of the International Symposium. Montreal: Etudes Editions.

Manosevitz, M., Prentice, N. W., \& Wilson, F. (1973). Individual family correlates of imaginary companions in preschool children. Developmental Psychology, 8, 72-79.

Marcia, J. E. (1966). Development and validation of ego identity status. Journal of Personality and Social Psychology, 3, 551-558.

Marcia, J. E. (1980). Identity in adolescence. In J. Adelson (Ed.), Handbook of adolescent psychology. New York. Wiley.

Matteson, D. (1975). Adolescence today: Sex roles and the search for identity. Homewood, IL: Dorsey Press.

Muus, R. E. (1980). Theories of adolescence (4th ed.). New York: Random House.

Reiff, A. J. (1961). Occupations and social status. New York: Free Press.

Somers, J. U., \& Yawhey, T. D. (1984). Imaginary play companions: Contributions of creative and intellectual abilities of young children. Journal Creative Behavior, 18, 77-89.

Spence, J., \& Helmreich, R. (1978). Masculinity and femininity: Their psychological dimension, correlates and antecedents. Austin: University of Texas Press.

Winnicott, D. W. (1965). The maturational processes and the facilitating environment. New York: International Universities Press.

Winnicott, D. W. (1971). Playing and reality. New York: Basic Books. 